\title{
Parametric Study on Wide Area Augmentation System for GPS Accuracy Enhancment
}

\author{
Dina Zayan \\ The British University in Egypt \\ Department of Electrical Engineering \\ El Sherouk City
}

\author{
Mohamed Rehan \\ The British University in Egypt \\ Department of Electrical Engineering \\ El Sherouk City
}

\begin{abstract}
GPS systems are used extensively for location determination in outdoor and urban areas. Many location-based services based on GPS system were introduced to the market over the previous few years. The GPS system was found to be of considerable limited accuracy due to several reasons which cause the accuracy to be in a range of 4 meters. Due to this, several accuracy enhancement techniques were proposed and some of them were standardized and deployed. The Wide Area Augmentation System (WAAS) is one these techniques which was developed in order to improve the GPS signal accuracy, availability and integrity. This paper focuses on the WAAS parameters and their performance in different environmental conditions that could cause ionospheric, satellite clock, and ephermis errors which consequently degrade the accuracy of the GPS signal. The WAAS architecture, performance, and main parameters are presented and their performance is simulated and analyzed under storming conditions. In addition, the potential of using the WAAS in combination with augmentation systems is discussed.
\end{abstract}

\section{General Terms}

Performance, Reliability, Verification, Experimentation.

\section{Keywords}

GPS accuracy, WAAS, satellite communications.

\section{INTODUCTION}

The Global Positioning System (GPS) [1-2] aims at providing reliable positioning, navigation, and timing services to worldwide users on a continuous basis in all weather, day and night, anywhere on Earth. GPS is made up of three segments; space segment, ground segment, and user segment. The space segment is represented with the satellite constellation sending the navigation and ranging data. The control segment (CS) monitors health and signal integrity and maintains the space vehicles (satellites) in their orbital configuration. Furthermore, the CS updates the satellite clock and ephermis corrections. Finally, the user receiver equipment (i.e., user segment) performs the navigation, timing, or other related functions.

GPS position determination is based on the concept of triangulation which is highly affected by the satellite geometry and the number of satellites from the receiver's view. Position determination also depends on the navigation and ranging data transmitted by the satellites. The satellites send these data modulated by a carrier frequency. Two frequencies are used for GPS signal transmission; L1 (1575.42 MHz) for civilian use, and the L2 (1227.60 MHz) for military use. Lately, a new civil signal L5 $(1176.45 \mathrm{MHz})$ is introduced to the system. The frequencies where chosen so as to avoid using beam antennae, avoid enormous ionospheric delays, and finally have the most immunity against changing weather effects. Unfortunately, current GPS signal accuracy ranges from 4-20 meters which is unacceptable in the aviation environment when translated in terms of VPL and availability measures.

This paper is organized as follows: Section 2 discusses the GPS outdoor signal error sources and accuracy limiting factors. Section 3 presents the WAAS system architecture [3-5] and expected performance. Section 4 provides simulation and analysis results as well as performance evaluation for the proposed system under different applied scenarios. Conclusions are presented in section 5 .

\section{GPS ACCURACY LIMITING FACTORS}

GPS signal accuracy for position determination is affected by many factors such as satellite geometry, ionospheric delays, tropospheric delays, satellite clock drifts, satellite ephermis errors, and finally receiver noise and multipath errors. The most significant accuracy degradation sources are those caused due to ionospheric delays, satellite's atomic clocks experiencing noise and clock drift errors [8-9].

These error sources lead to unacceptable accuracy measures in the aviation environment. The safety-of-life operations in this environment require highly accurate position calculations as well as high integrity measures on these calculations.

Differential GPS (DGPS) represents a means for providing an improvement for the positioning or timing performance of GPS using reference stations at fixed known locations, each equipped with at least one GPS receiver. The idea of differential corrections is that the reference station always has a known fixed position. Since the actual positions of the satellites and the receiver are known (i.e. the true range/actual distance is known). The distance calculated by the receiver using the normal GPS broadcast signals is known as the pseudo-range, which is generally in error due to the combined error sources. The difference between the true range and the pseudo-range is the error and is known as the differential correction or the error factor. This factor is then sent to the local GPS receivers in order to apply the necessary corrections as shown in Figure 1. DGPS can bring the accuracy of the readings down to 1-3 meters of the object, as compared to 4-20 meters of the normal GPS signal. 


\section{Differential GPS}

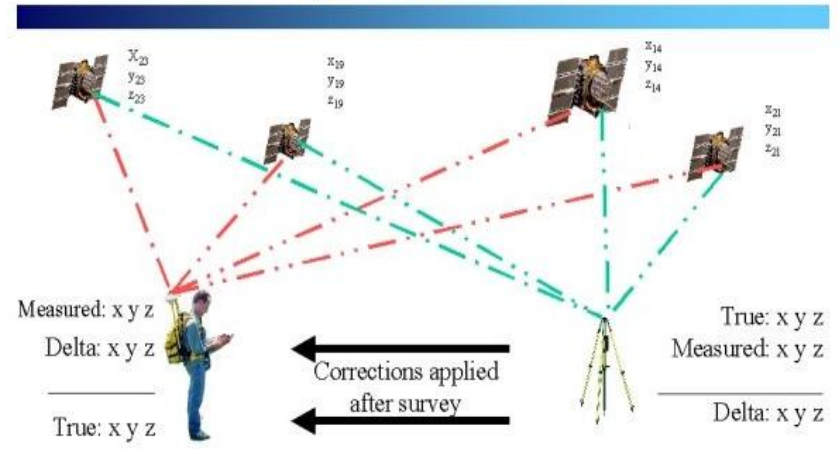

Fig 1: Differential Correction Concept.

The DGPS The reference stations of the DGPS provide information to the users via a data link that may include:

- Corrections to the end user's pseudo-range measurements, and corrections to the satellite clock or ephemeris data.

- Raw reference station measurements (such as pseudorange or the carrier phase).

- Integrity data; such as the "use" and "don't use" indicators for each visible satellite.

- Auxiliary data including the health, location, and any other required data from the reference stations.

The Wide Area DGPS (WADGPS) network consists of one master station, a number of monitor stations, and a communication link. Each monitor station is equipped with a high quality rubidium clock and a GPS receiver capable of tracking all satellites within the field of view. GPS measurements taken at each reference station are sent to the master station. The master station estimates Ionospheric time delay parameters, calculates the satellite ephermis and clock errors based on the known location of the monitor station and the information collected. The computed error corrections are transmitted to the users via a convenient communication link such as a satellite.

The proposed monitor station computation process is shown in Figure 2. As mentioned previously, each monitor station is equipped with a clock, sensor, and a receiver that are capable of providing error measurements. The Ionospheric errors [8], corrected tropospheric errors, and the pseudorange measurements are sent together to the master station. The tropospheric errors are corrected in the monitoring station based on a tropospheric delay algorithm which involves several readings such as the temperature, humidity, and pressure readings from the monitor station sensors.

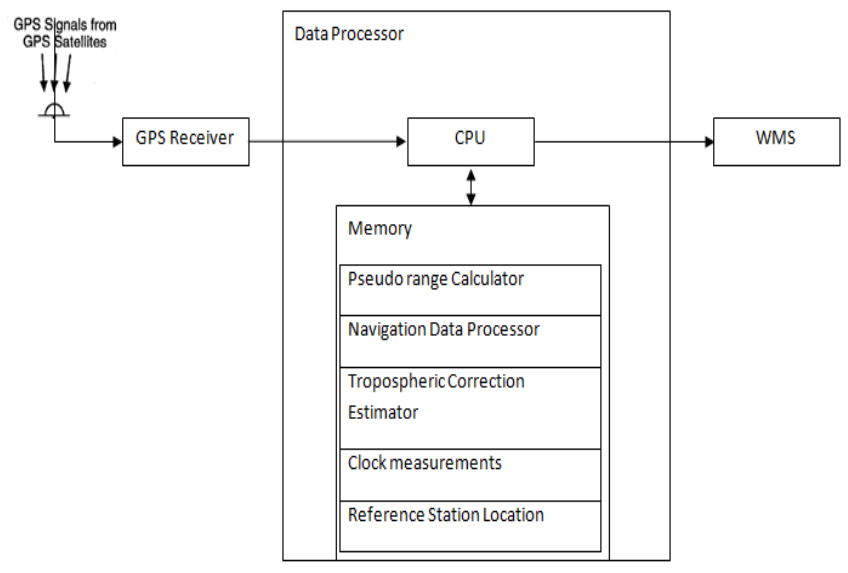

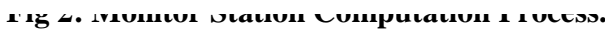

The proposed master station computation process is shown in Figure 3. The master station is in continuous communication with all of the reference stations. The primary function of the master station is to receive the computed pseudo range measurements from all the monitoring stations in order to be able to estimate the ephermis errors, satellite clock errors, monitor stations receiver clock offsets, and to transmit these error corrections to the users. Hence it comprises a pseudo range residual synchronizer, ephermis and clock correction estimator for each GPS satellite observed by the reference station, and a transmitter.

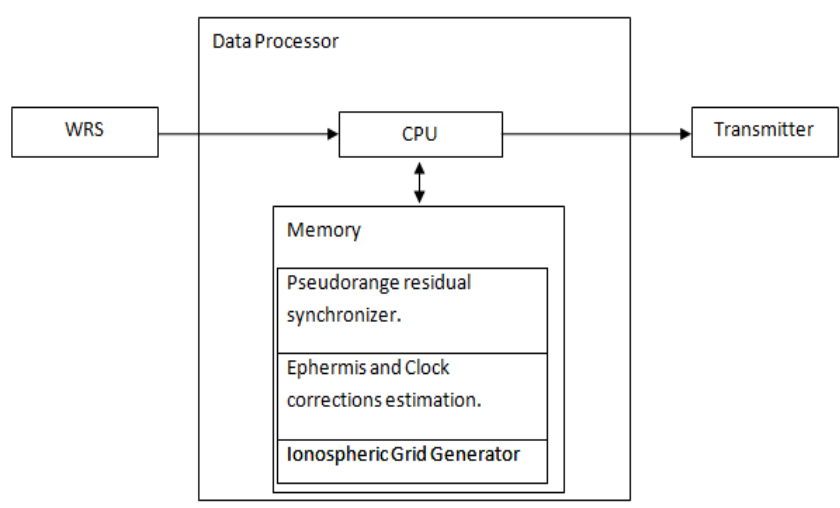

Fig 3: Master Station Computation Process.

A typical user who would benefit from the WADGPS would be equipped with a GPS receiver and a quartz oscillator. Each user collects pseudo range(s) along with the ephermis data for all the GPS satellites in view. The user also receives the error correction vector sent by the master station via the appropriate communication link (which is normally the satellite communication link). These corrections are then applied to the user's raw measurements, and the adjusted ones are then used.

\section{WAAS ENHANCEMENT}

The WAAS utilizes the concept of the Wide Area Differential GPS in terms of calculating an error factor by knowing the true position and the pseudorange. The concept of operation could be summarized as follows:

1. WAAS reference stations are placed all over the United states in order to measure the pseudo ranges and the carrier phases for both GPS signal frequencies L1, and L2 (also 
being improved to include measurements for L5 frequency).

2. The WAAS reference stations send their measurements to the WMS which performs calculations on these measurements. These calculations include the clock and ephermis corrections for each GPS satellite, ephermis for each GEO satellite and finally the Ionospheric delays on the ionospheric grid and their corrections. The grid (imaginary Ionospheric thin shell) is composed of ionospheric grid points (IGPs) separated by $5^{\circ} * 5^{\circ}$ at an altitude of $350 \mathrm{~km}$ above the Earth's surface.

3. In addition to the calculated corrections, the WMS also calculates error bounds. The error bounds for ionospheric corrections are called Grid Ionospheric Vertical Errors GIVEs - at each IGP, and also the error bounds for clock and ephermis corrections for each GPS satellite in view, are called the User Differential Range Errors (UDREs).

4. The WMS sends both the corrections and the error bounds to the users via the GEO communication satellites.

5. Users use the corrected range measurements to compute their positions. They also apply the results from the error bounds (GIVEs and UDREs) on their computed positions to ensure accuracy. The resulting position bounds are called the Horizontal Protection Level (HPL), and the Vertical Protection Level (VPL); these are used to determine whether to proceed with certain flight operations.

For precision approach (PA) flight operations -which is our focus in this paper -, Probability (VPL $\leq$ VAL, and HPL $\leq$ HAL) $\geq X$, Where $0.95 \leq \mathrm{X} \leq 0.99$, and $\mathrm{X}$ differs according to the WAAS phase.

\section{ANALYSiS AND Simulation}

In this section, we'll examine the important parameters of the WAAS system and their effect on the requirements of WAAS in terms of minimum of $99 \%$ vertical and horizontal accuracy, integrity risk (VAL, HAL), continuity and availability. Our simulation was done using the Matlab Algorithm Availability Simulation Tool (MAAST) developed by Stanford University. The basic GEOs that are used in our simulations are the PRN135 and the PRN-138. The following parameters were used to measure WAAS system performance in flight operations:

- Availability contour.

- VPL contour

We wanted to study the effects of different parameters on enhancing the WAAS signal accuracy and availability in terms of the previously mentioned measures. First, in order to observe the effect of the reference stations and expanding the IGP mask on the WAAS availability, we simulated the VPL contour (which is used to determine the service availability) using the Initial Operational Capability (IOC) requirements which are as follows:

1. 24 standard GPS satellite constellation (by selection MOPS from the WAAS control panel) defined in the WAAS MOPS.

2. User grid should be $1^{\circ}$

3. Thirty second time step over a 24 hour simulation period.

4. 25 reference stations.

5. MOPS operating mode with precision approach (PA) option.

6. Use the MOPS_UDRE and MOPS_GIVE defined algorithms which ensure VHPL information integrity for the VPL and the HPL calculation.
7. UDRE and GIVE algorithms are replaced by constant values where $\mathrm{UDRE}=1.25$ and the GIVE $=1.2$.

8. Use the number of IGPs $=190$ point.

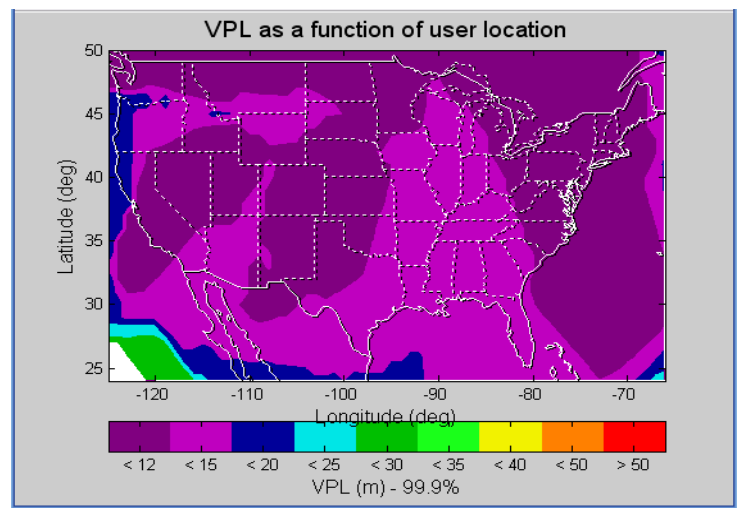

Fig 4: Simulated VPL using constant GIVE and UDRE values.

\subsection{Increasing the Number of Reference Stations and IGP}

Since the IOC requirements, WAAS has been upgraded, where 13 new reference stations have been added to the ground network. The ground stations have been added in Mexico, Canada, and Alaska. This is intended to enhance the WAAS service availability as will be shown below. The effect of increasing the number of reference stations is observed mainly when calculating corrections and the error bounds using GIVE and UDRE algorithms in the master station. The ionospheric correction operating principal depends on employing reference stations at known positions to monitor the GPS signals to be able to calculate corrections/error factor. The key to the success of this approach is to have the number of reference stations and ionospheric grid points large enough in order to account for the ionospheric variations. The correction estimates are done on IPPs (Ionospheric Pierce Point) where the signal crosses the IGPs on the IGP mask -where the reference station/receiver is located-. Thus, expanding the IGP mask will enhance the availability only when accompanied by increasing the number of reference stations, and vice versa and both effect the GIVE value.

The upgraded WAAS architecture with 38 reference stations with an expanded IGP mask around Canada and Alaska was used, but with all the other previous parameter used. We can see more coverage (Figure 5) but without enhancement in the middle region. This is because the reference stations weren't added in the same area, but were used in order to expand the availability rather than enhance the VPL in the same regions

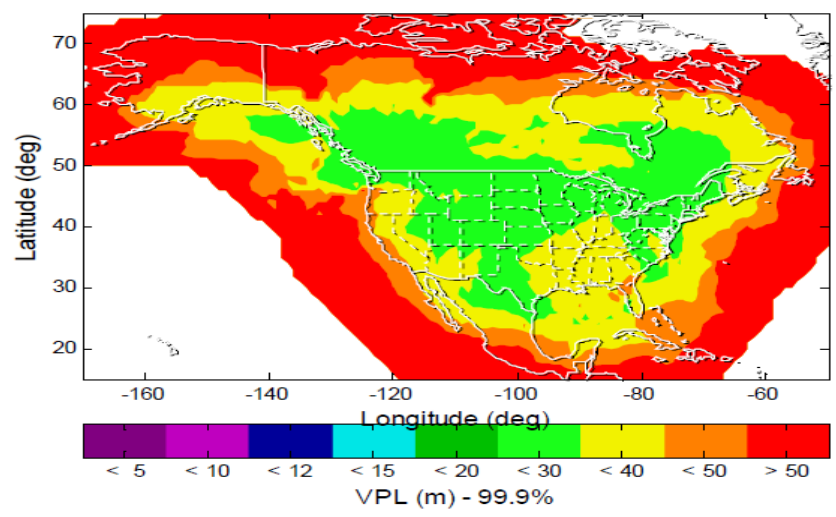

Fig 5: Simulated VPL using the upgraded WAAS architecture. 


\subsection{Effect of use of GEO Data on Accuracy}

As shown in Figure 6, increasing the number of GEOs in the simulation, leads to some availability improvement (in terms of better VPL, i.e. VPL $<50$ meters has improved for $99.9 \%$ of the time), since we ensured that 2 GEOs are always observed by the north location. Since the GEOs provide their own ranging signals, thus this might improve the satellite geometry for certain locations aiming at continuity and better position estimates.

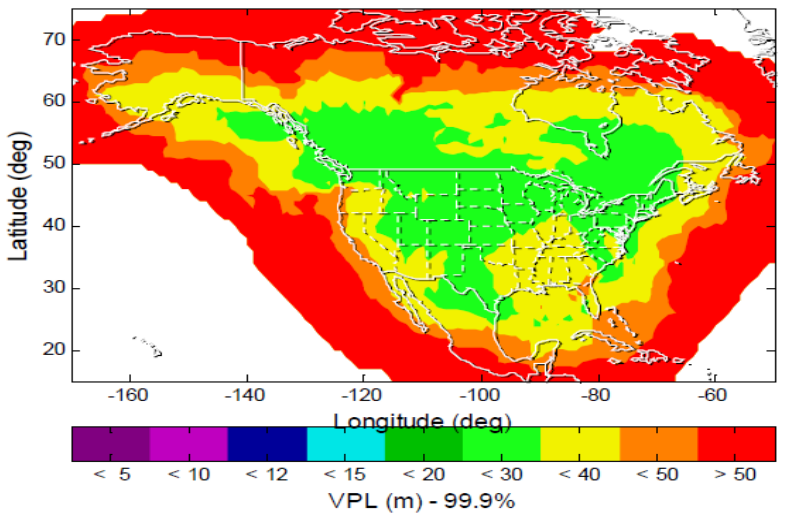

Fig 6: VPL plot when adding a GEO satellite.

\subsection{WAAS Accuracy under Storming Conditions}

Based on the previous study, we could predict the performance of the WAAS System under extreme weather effects such as storms. Severe storms can cause large rate of change of the ionospheric delay as well as large spatial gradients. WAAS system should be designed so as to account for these changes as fast as possible since these changes would affect ionospheric corrections, GIVE values more often than in a normal weather condition. Both the GIVE and delay values will increase in the vicinity of a storm which would affect the protection levels and service availability.

Under normal conditions, the interval used to calculate the ionospheric delay is every 5 minutes. Under storm conditions, WAAS will calculate large delay and GIVE values at each IGP. Since the impact of large GIVE values is not preferable on the protection level bounds which the user depends on for providing integrity data, the system will start calculating the delay and GIVE values every 1 minute.

If we plot these values for both storm and non-storm conditions with real collected data measurements, we'll find that the residual errors and GIVE values are much higher for storm conditions which decrease the probability of the VPL and HPL being below their alert limits as shown in Figure 7

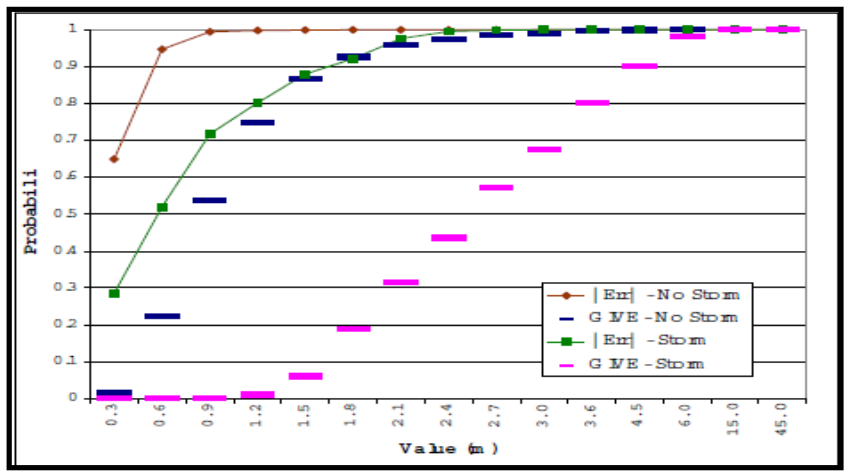

Fig 7: Storm conditions increase the error and GIVE values (values are read from $x$-axis)

\subsection{Use of Dual Frequency}

Finally, we would like to address a new approach in our analysis, which is the usage of dual civilian frequencies (both L1 and L5 frequency) and their effect on the WAAS systems.

In an attempt to address the largest delay estimation in the WAAS system (ionospheric delay), the need for better improvement of its error estimation and correction was raised. This need lead to the potential use of both L1 and L5 frequencies together which would improve the performance for GPS users by allowing them to estimate and correct/mitigate the ionospheric errors. This is done in a way through which the GPS dual frequency receiver would be directly involved in estimating the ionospheric delay and applying its error factor to the pseudorange measurements for each line of sight GPS satellite without any help needed from the WAAS system.

This approach will definitely decrease the uncertainty in the taken pseudorange measurements, and when used together with improving the remaining correction and confidence bound algorithms, this will enhance the performance of the WAAS [6], [7]. There is another major advantage from using the dual frequency system which is its relative immunity against unintentional interference. So if one of the signal frequencies has been jammed, we will still have visual and measurements from the second signal from the same satellite.

To determine their effect on the Vertical Protection Level (VPL) and availability measures in the aviation environment. This paper will first investigate the WAAS initial operational capability (IOC) performance effect on improving the VPL so that it won't exceed the alert limits, then an analysis and simulation for adding reference stations, increasing the Ionospheric Grid Point (IGP) mask, increasing the number of Geostationary communications satellites (GEOs), using L1-L5 dual frequency system.

Figure 8 shows the difference between the error components when making the WAAS calculations for both the single and dual frequency measurements. The dual frequency overall error is similar to the single frequency one with exception of that fact that it doesn't contain a term for ionospheric error corrections since the delays are directly mitigated at the receiver so it's combined with the. This term is of course smaller than the term that was included due to ionospheric corrections estimation.

As we can see, the term based on the UDRE is the dominant error component for the L1-L5 dual frequency usage. We can also observe the better overall estimation and performance with lower errors in the dual frequency than in the single frequency receivers. This would ensure more accurate VPL plots and hence better performance and availability for flight operation (where VPL $<20$ meters) for $99.9 \%$ of the time.

L1 Single Frequency User Error Bound

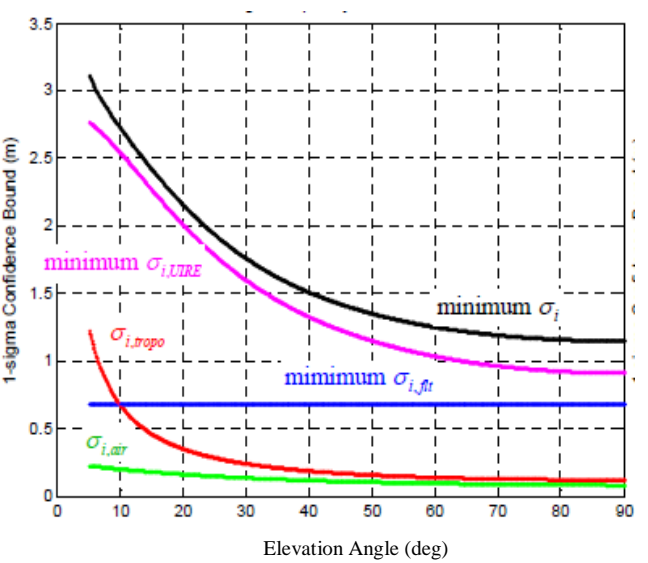


L1 L5 Single Frequency User Error Bound

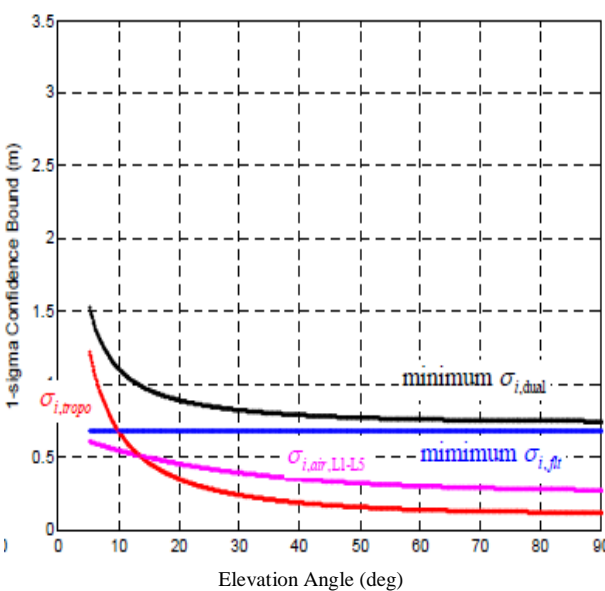

Fig 8, 9: Comparison between the error factors of single and dual frequency systems.

\section{ConcLusions}

In this paper the current GPS limitations and their effect on the GPS signal accuracy and integrity were presented. The WAAS, which is a differential GPS based system introduced to provide corrections on the most important GPS error sources, was described and its performance was analysis. Examples of these errors include the satellite clock and ephermis errors as well as the ionospheric corrections. We also verified that we could lower the VPL by expanding the IGP mask (i.e. increasing the number of IGPs) and hence improve the signal availability for $99.9 \%$ of the time. We also studied and were able to prove the effect of the GIVE and the UDRE values in the accuracy and integrity of the WAAS. The paper also discusses the proposed use of the second frequency signal (L5) and verified that it would provide a significant performance improvement.

\section{REFERENCES}

[1] Anis Drira, "GPS Navigation for Outdoor and Indoor Environment”, University of Tennessee, Knoxville, 2006.

[2] Elliott D. Kaplan, Christopher J. Hegarty, "Understanding GPS, Principles and Applications", second edition, ARTECH House, 2006.

[3] Jan, S.S.; Gebre-Egziabher, D.; Walter, T. Enge, P, "Improving GPS-Based Landing System Performance Using an Empirical Barometric Altimeter Confidence Bound”,. IEEE T. Aero. Elec. Sys., 44, 127-146, 2008.

[4] Jan, S.S, "Aircraft Landing Using a Modernized Global Position System and the Wide Area Augmentation System", $\mathrm{Ph} . D$. thesis, Department of Aeronautics and Astronautics, Stanford University, USA, 2003.

[5] Todd Walter and Per Enge, "System Overview, Recent Developments, and Future outlook for WAAS and LAAS", Stanford University, 2004.

[6] "Global Positioning System, Wide Area Augmentation System Performance Standard", Federal Aviation Administration, $31^{\text {st }}$ October 2008.

[7] J. David Powell, "Space Weather: It's Effect on GNSS DGNSS, SBAS, and Flight Inspection", Aero/Astro Dept., Stanford University, Stanford, CA, 2010.

[8] S.S.Riaz Ahamed, "The Effects of Global Positioning System for Reliable Positioning, Navigation, and Timing Services", Journal of Theoretical and Applied Information Technology, 2008.

[9] "Vertical Guidance Performance Analysis of the L1-L5 Dual Frequency GPS/WAAS User Avionics Sensor", Institute of Civil Aviation, Shau Shiun Jun, 25 March 2010.

[10] Todd Walter and Per Enge, "Modernizing WAAS", Stanford University, 2005. 